\title{
Canadian funders and institutions are lagging on reporting results of clinical trials
}

\author{
Kelly D. Cobey PhD, Dean Fergusson PhD, David Moher PhD
}

Cite as: CMAJ 2017 October 23;189:E1302-3. doi: 10.1503/cmaj.170840

A ccumulating evidence suggests that the results from many clinical trials are not shared. A systematic review that examined trials approved by research ethics committees or identified in clinical trial registries found that about 50\% did not report their results. ${ }^{1}$ This review also found that studies with statistically positive findings were more likely to have had results reported. A globally important example of the potential public health and fiscal consequences of failing to report trial results is that of the antiviral drug oseltamivir, which was purchased, stockpiled and used globally for the treatment and prevention of seasonal and pandemic influenza based on biased study reporting and incomplete evidence. ${ }^{2}$ Canadian regulators, research institutions and funders must act alongside others internationally to ensure that registration, and complete and transparent reporting of clinical trials become an important global priority.

Several positive actions have occurred that address improvements in both trial registration and results reporting in registries. In the 2013 Declaration of Helsinki, the World Medical Association explicitly stated that clinical trials must be registered and have their study results reported. ${ }^{3}$ The AllTrials campaign was also launched in 2013. A related petition for all trials to be registered and reported has since garnered more than 90000 signatures from individuals and more than 700 signatures from organizations. ${ }^{4}$ In 2015 , the World Health Organization (WHO) reiterated its stance on the importance of registering and reporting trial results. The WHO specifies a 12-month deadline for results to appear in registries. ${ }^{5}$ On May 18, 2017, nine major research funders across the globe released a joint statement via the WHO International Clinical Trials Registry Platform on public disclosure of results from clinical trials. The nine funders affirmed the importance of sharing clinical trial results in a timely fashion, and they pledged to implement policy and impose audits to ensure that trials they support are appropriately reported. ${ }^{5}$ Regrettably, no publicly funded health research agency in Canada was named in the recent statement.

Does silence on the matter from Canadian funders send a message to the Canadian research community that they do not value the reporting of trial results? This would be out of step with the community of global funders. We strongly encourage Canadian funders of research to step up to the plate and sign on to the joint statement. Funders have an obligation to patients participating in research, the public and research professionals to promote and uphold international best practices pertaining to clinical trials.

\section{KEY POINTS}

- Researchers, research institutions, health funders and regulators have an obligation to patients to ensure clinical trials are registered and have their results reported in trial registries.

- Canadian health funders and regulators should commit to upholding the international joint statement on public disclosure of clinical trial results.

- Canadian health funders, regulators and research institutions should audit and enforce timely registration and results reporting of clinical trials in trial registries.

- Dedicated training to support Canadian researchers in achieving best practices in trial registration and reporting should be developed collaboratively by stakeholders.

Another way to promote change in Canada would be to require explicit registration of clinical trials and results reporting, with firm enforcement of implementation of such policies via routine audits by institutions and funders. Although the Tri-Council Policy Statement: Ethical Conduct for Research Involving Humans (i.e., TCPS 2) devotes an entire chapter to clinical trials, the framework does not provide explicit guidance for trial reporting. For example, it states that [where possible] clinical trial results should be reported in the registry where they were initially registered "in a timely manner without undue restriction" (Article 11.12). ${ }^{6}$ This falls short of other international standards that specify firm timelines. ${ }^{5}$ The lack of detail provides little structure for institutions (e.g., universities or hospitals) to enforce compliance, and requires the development and mandate of unnecessary independent institutional policies. Health Canada's requirements for the public release of clinical trial information has similar shortcomings (i.e., timelines for reporting are not specified). Furthermore, the policy language contains ambiguity that provides a mechanism through which regulators may withhold data (e.g., claiming the study is ongoing when it is not). ${ }^{7}$

The TCPS 2 document also explicitly notes:

If research findings and the research materials and research data they are based upon, are not disseminated (e.g., published in a peer-reviewed journal, added to a publicly available clinical trials database) within a reasonable time, their value may be diminished or lost, betraying the contributions and sacrifices of participants. ${ }^{7}$ 
This is an important sentiment and has been echoed by patient advocacy groups who hold that clinical trial registration and results reporting are important and necessary steps to increasing the value of health research. Why should patients participate in clinical trials if their contribution is not valued (i.e., trial results are not reported)? Similarly, in our Canadian health care system where tax revenues help fund research, it is unclear whether use of such monies should be used for trials that go unreported.

In the current Canadian context, linking a publication to a clinical trial results registry may be regarded as sufficient reporting. Although this is better than nonsharing altogether, simply linking publications to registry records is suboptimal. First, we know that many published trials fail to report their study outcomes clearly and completely, with outcome switching and removal being all too prevalent. ${ }^{8}$ Trial registration and the availability of a full study protocol are essential for auditing these behaviours. Publications linked to registries are not audited. Second, many published manuscripts are published behind paywalls, therefore, linking articles to trial registries will not provide access to all. One benefit of reporting trial results in the registry where the trial results were first registered is that it provides an immediate, free and standardized open access account of the study.

Ensuring that trial results are reported is a shared responsibility. Institutions that employ researchers and facilitate clinical trials have often been in the backgrounds of these discussions. Linking best practices at Canadian research institutions to broader initiatives led by national and international stakeholders will be critical to achieving progress. For example, the newly developed TrialsTracker tool allows research institutions (with more than 30 trials) to audit their trial reporting. ${ }^{9}$ Although this tool has limitations, ${ }^{10}$ it provides a useful starting point for audit and feedback; it also has a low barrier for implementation. Furthermore, institutions are obligated to support their researchers in the reporting of their trials, but it is unclear if such support is commonplace.

The Centre for Journalology at the Ottawa Hospital Research Institute has added a publications officer (K.D.C.), a role that provides institutional support and educational outreach to researchers as they navigate the research-to-publication process and to ensure best practice. ${ }^{11}$ The centre is leading an internal audit and has contacted all researchers who have outstanding trial results and requested that they complete their registry records. If all institutions invested in similar roles and audits, resources and outreach materials could be developed and shared publicly to prevent duplication of effort and reduce costs. Networks could be established to share best practices. Efforts by institutions to provide support are likely to be well received by researchers who may have difficulty keeping pace with various mandates concerning research ethics, design, conduct and reporting requirements related to trials. Institutional committees for promotion and tenure need to provide stronger incentives and rewards to faculty for registering their trials and subsequently reporting the completed results.

Broad failure to register and to report trials transparently has been well documented. The incentives to publish any aspect of research work in a journal with a high impact factor, and to do so quickly, are greater than the incentives to share clear and complete accounts of study results promptly in publicly available registries. We also need to rethink what it means to "publish" research and what information is needed to do so efficiently and transparently.

Canadian funders, regulators, institutions and researchers should seek to be leaders in the global movement to remedy reporting problems, and act proactively and collaboratively to develop solutions and monitoring frameworks to address this issue. All Canadians would benefit from such actions.

\section{References}

1. Schmucker C, Schell LK, Portalupi S, et al. Extent of non-publication in cohorts of studies approved by research ethics committees or included in trial registries. PLoS One 2014;9:e114023.

2. Jefferson $T$, Jones $M$, Doshi $P$, et al. Neuraminidase inhibitors for preventing and treating influenza in adults and children. Cochrane Database Syst Rev 2014;(4):CD008965.

3. World Medical Association. World Medical Association Declaration of Helsinki: ethical principles for medical research involving human subjects. JAMA 2013;310:2191-4.

4. AllTrials campaign petition. London (UK): Sense About Science. Available: www.alltrials.net/petition/ (accessed 2017 June 5).

5. WHO statement on public disclosure of clinical trial results. Geneva: World Health Organization; 2015. Available: www.who.int/ictrp/results/reporting (accessed 2017 June 5).

6. Tri-Council policy statement: ethical conduct for research involving humans. Ottawa: Secretariat on Responsible Conduct of Research; 2014. Available: www.pre .ethics.gc.ca/pdf/eng/tcps2-2014/TCPS_2_FINAL_Web.pdf (accessed 2017 Aug. 17).

7. Public release of clinical information in drug submissions and medical device applications. Ottawa: Health Canada; 2017. Available: www.canada.ca/content/dam/ hc-sc/documents/programs/public-release-clinical-information-drug-submissions -medical-device-applications.pdf (accessed 2017 Aug. 17).

8. Chan AW, Krlez K, Schmid I, et al. Outcome reporting bias in randomized trials funded by the Canadian Institutes of Health Research. CMAJ 2004;171:735-40.

9. Powell-Smith A, Goldacre B. The TrialsTracker: automated ongoing monitoring of failure to share clinical trial results by all major companies and research institutions [ version 1] F1000 Res 2016;5:2629.

10. Coens, C, Bogaerts J, Collette L. Comment on the "TrialsTracker: Automated ongoing monitoring of failure to share clinical trial results by all major companies and research institutions." [version 1; referees: 1 approved, 1 approved with reservations] F1000 Res 2017;71:1-14.

11. Cobey KD, Galipeau J, Shamseer L, et al. Report on a pilot project to introduce a publications officer. CMAJ 2016;188:E279-80.

Competing interests: Kelly Cobey is employed as the Publications Officer for the Centre for Journalology at the Ottawa Hospital Research Institute. No other competing interests were declared.

This article has been peer reviewed.

Affiliations: Centre for Journalology, Clinical Epidemiology Program (Cobey, Fergusson, Moher), Ottawa Methods Centre, Ottawa Hospital Research Institute; School of Epidemiology, Public Health, and Preventative Medicine (Cobey, Fergusson, Moher), Faculty of Medicine, University of Ottawa, Ottawa, Ont.; Department of Psychology (Cobey), University of Stirling, Stirling, UK

Contributors: Kelly Cobey drafted the original manuscript. David Moher and Dean Fergusson made substantial contributions to the manuscript. All of the authors revised the manuscript critically for important intellectual content, gave final approval of the version to be published and agreed to be acccountable for all aspects of the work.

Funding: This work was unfunded. David Moher holds a University of Ottawa Research Chair.

Correspondence to: Kelly Cobey, kcobey@ohri.ca 